\title{
Analysis of Inter-Satellite Terahertz Communication Link
}

\author{
Yuan-Ming Ding, Shan Gao, Xin Shi, Hao Wu \\ Communication and Network Key Laboratory, Dalian University, Dalian Liaoning, China \\ E-mail: dingyuanming@dlu.edu.cn
}

\begin{abstract}
The domestic and foreign status of terahertz wireless communication is introduced, and the paper analyzes the feasibility of ultra high speed short range and high speed long range inter-satellite terahertz communication. The capacity of inter-satellite terahertz communication system under the ideal additive white Gaussian noise (AWGN) channel is studied. It simulates the inter-satellite terahertz communication link and calculates high gain antenna design parameter requirements by analyzing more than $100 \mathrm{Gbps}$ capacity of short range inter-satellite communication and more than $2.5 \mathrm{Gbps}$ capacity of long range inter-satellite communication.
\end{abstract}

Keywords-satellite communication; terahertz communication; inter-satellites link

\section{INTRODUCTION}

Terahertz wave has many features, such as wide frequency band, high speed, small scattering, high penetrability, good directionality, high security and so on. It is another communication frequency band between microwave communication and laser communication. In recent years, the development of terahertz communication technology has become the hot topics in the study of high speed large capacity communications in developed countries. China, Japan, Germany, France, the United States, Canada and other countries also successfully carried out terahertz communication system research and experiment.

\section{A. Japan}

In 2004, Japan's NTT company for practical application of terahertz wireless communication system, in $0.12 \mathrm{THz}$ carrier, realized the remote (more than $1 \mathrm{~km}$ ) communication in 6HDTV signals $10 \mathrm{Gbps}$ wireless transmission link. In 2012, the company set up $0.3 \mathrm{THz}$ wireless communication demonstration system, using the amplitude shift keying (ASK) modulation way, realized the short distance $(0 \sim 0.5 \mathrm{~m})$ 24Gbps error-free transmission (bit error rate is better than ten of negative nine power)[1].

\section{B. Germany}

In 2011 year, Germany's Fraunhofer institute for applied solid state physics (IAF) designed $0.3 \mathrm{THz}$ wireless communication system in which the modulation systems were ASK and BPSK. When transmission distance was $5 \mathrm{~m}$, transmission rate was $10 \mathrm{Gbps}$. In the same year, IAF set up $0.22 \mathrm{THz}$ wireless communication demonstration system, when the output power is about $1.4 \mathrm{~mW}$, the institute adopted 16/64 /128/256QAM modulation system, which conducted a $2 \mathrm{~m}$ transmission distance, $12.5 \mathrm{Gbps}$ experiment, and completed the pure atmosphere, heavy rain and heavy fog weather conditions of $\mathrm{THz}$ wave attenuation testing [2]. In 2012 year, the system was improved, which conducted $20 \mathrm{~m}$, $15 \mathrm{Gbps}$ and $10 \mathrm{~m}, 25 \mathrm{Gbps}$ communication demonstration experiments. In 2013 year, when the communication experiment distance was $1 \mathrm{~km}$, transmission rate reached $40 \mathrm{Gbps}$, implemented on communication capacity and the seamless connection of optical fiber communication [3]. The physikalisch technische bundesanstalt (PTB) and the federation technische bundesanstalt (FTB) built a transmitting and receiving modules independent system used in $0.3 \mathrm{THz}$ measurement and transmission system of communication channel experiment.

\section{China}

In 2011 year, China academy of engineering physics (CAEP) developed a set of $0.14 \mathrm{THz}$ communication system based on the structure of super-heterodyne. It adopted 16QAM advanced digital modulation, completed the $500 \mathrm{~m}$ and 10Gbps experimental verification [4]. In 2013 year, the CAEP also developed a set of $0.34 \mathrm{THz}$ system based on the SSB modulation system, in which the transmission rate was $3 \mathrm{Gbps}$ and transmission power was $14.58 \mathrm{dBm}$, but it can only achieve $0.25 \mathrm{~m}$ short distance transmission [5]. In 2013 year, the China academy of sciences Shanghai micro system set up a $3.9 \mathrm{THz}$ system with the communication rate of $1 \mathrm{Mbps}$ which achieved $2.4 \mathrm{~m}$ video wireless transmission. In 2015 year, it also carried out wireless communication system research based on terahertz quantum cascade lasers (QCL) and completed the real-time video transmission experiment [6].

In addition, the city university of Hong Kong and university of electronic science and technology also designed terahertz communication experiment systems. Currently, the transmission distance of the before-mentioned terahertz communication experiment system is within $1 \mathrm{~km}$, mostly confines to the indoor short distance transmission experiment.

\section{Other Countries}

In 2009 year, the university of Toronto set up $0.14 \mathrm{THz}$ communication demonstration system using ASK modulation system and proceeded the short distance $(0 \sim$ $1.15 \mathrm{~m}$ ) of 4Gbps transmission experiment [7]. In 2011 year, the United States Bell laboratory set up a $0.625 \mathrm{THz}$ communication demonstration system, used for short distance $(0 \sim 0.5 \mathrm{~m})$ transmission experiment, when the output power was about $1 \mathrm{mw}$, transmission rate was $2.5 \mathrm{Gbps}$ [8]. In 2015 year, Lille university completed the terahertz wireless data link experiment, which is $0.3 \mathrm{THz}$ working frequency, and $25 \mathrm{~m}$ communication distance [9].

Reference [10] is on the background of long distance satellite terahertz communication applications, it simulates and analyzes transmission performance of inter-satellite communication link. It is concluded that the electron 
emission system has a higher signal to noise ratio (SNR) and lower error rate than photoemission system, which can choose BPSK and 16QAM modulation system for variable rate transmission. Nevertheless, when the communication distance between the satellite increases to $100 \mathrm{~km}$, space transmission loss reaches hundreds of $\mathrm{dB}$. This suggests that the long distance terahertz communication link need higher antenna gain. Therefore, it is necessary to further analyze the feasibility of terahertz satellite long distance communication for terahertz communication applications to explore new ways. At the same time, in the design of launch system of inter-satellite terahertz communication, we also must consider the space transmission characteristics of terahertz wave through link design to compensate for transmission loss.

Reference [11] researches potential space applications of the terahertz wireless communication system, through the analysis of wireless communication link about spacecraft indoor WLAN and the planet's surface wireless communication application. It is concluded that each $1 \mathrm{GHz}$ channel bandwidth can be obtained maximum data rate more than $10 \mathrm{Gbps}$, pointing out that the high gain antenna and high power transmitter used in long distance communication between satellites is feasible. Terahertz spectrum of state-of-the-art antenna technology can reduce the design cost and improve the system performance, higher antenna gain can also increase the system capacity. References [12] and [13] separately designed the high gain reflective antenna system and mechanical scanning confocal elliptical antenna system. However, with the increasing antenna gain and terahertz frequency, narrow beam width for terahertz applications is a challenge, because it needs antenna tracking and locating accurately.

Long range between the satellites of the large capacity terahertz communication need high gain antenna and high power launch system, at the same time, also need acquisition pointing tracking (APT) subsystem safeguard. This paper, based on the existing high gain reflective antenna, analyzes capacity of inter-satellite terahertz communication system. It calculates satellite communication parameters of high gain antenna design requirements which are more than 100Gbps high capacity, short distance and $2.5 \mathrm{Gbps}$ high capacity, long distance.

\section{TERAHERTZ SATELLITE COMMUNICATION SYSTEM CAPACITY}

When terahertz satellite communication system is assumed to be the additive white Gaussian noise (AWGN) channel, the system capacity can be calculated by the Shannon theorem:

$$
C=B \log _{2}(1+S N R)
$$

In the formula, SNR is signal to noise ratio, $\mathrm{B}$ is channel bandwidth. SNR's formula is:

$$
S N R=\frac{P_{R}}{F k T B}
$$

In the formula, $P_{R}$ is received power, $\mathrm{K}$ is Boltzmann constant, $\mathrm{T}$ is environment temperature, $\mathrm{F}$ is additional noise receiver.

According to the electromagnetic wave transmission model, system's received power $P_{R}$ is:

$$
P_{R}=\frac{P_{r} \eta_{r} \eta_{R} G_{r} G_{R}}{L_{f}}
$$

In the formula, $P_{T}$ is transmitter power, $G_{T}$ and $G_{R}$ separately are transmitting antenna and receiving antenna gain, $L_{f}$ is free space transmission loss, $\eta_{T}$ and $\eta_{R}$ separately are transmitting antenna and receiving antenna efficiency. When the integration of the sending and receiving antenna is used, the efficiency of the antenna is $\eta_{T}=\eta_{R}$, antenna gain is:

$$
G_{T}=G_{R}=\left(\frac{\pi D_{a}}{\lambda}\right)^{2}
$$

$\lambda$ is wavelength, $D_{\text {a }}$ is antenna diameter, $L_{f}$ is free space propagation loss.

$$
L_{f}(d B)=10 \lg \left(\frac{4 \pi d}{\lambda}\right)^{2}
$$

Here $\mathrm{d}$ is receiving distance, the unit is $\mathrm{km}$

Hence, without considering the satellite platform vibration effect, system power for the receiver to receive is:

$$
\begin{aligned}
P_{R} & =P_{T} \eta_{T}^{2}\left[\pi\left(\frac{D_{a}}{2}\right)^{2}\right]^{2}\left(\frac{f}{c d}\right)^{2} \\
& =P_{T} \eta_{T}{ }^{2} \pi^{2} \frac{D_{a}^{4} f^{2}}{16 c^{2} d^{2}}
\end{aligned}
$$

When $C_{0}=\sqrt{\eta_{T}^{2} \pi^{2} / 16 c^{2}}$, the above formula can be
onverted to:

$$
P_{R}=P_{T} \eta_{T} \frac{C_{0}^{2} D_{a}^{4} f^{2}}{d^{2}}
$$

When formula (2) and (7) substitute into formula (1), terahertz satellite communication link which is calculated under ideal AWGN channel can reach the maximum capacity. 


\section{TERAHERTZ SATELLITE COMMUNICATION LINK SIMULATION ANALYSIS}

In the following simulation analysis and calculation, the assumed parameters are as follows: $0.34 \mathrm{THz}$ frequency, bandwidth of $10 \mathrm{GHz}$ and $50 \mathrm{GHz}$ respectively, choice of transmitted power of $10 \mathrm{mw}$ and $1 \mathrm{w}$ respectively, $300 \mathrm{~K}$ environment temperature.

\section{A. Short Range Satellite Communication}

When the satellite communication distance is within $1 \mathrm{~km}$, maximum communication capacity of terahertz communication system is shown in Fig.1 and Fig.2. Figure 1 is the maximum capacity of the short distance communication system, communication frequency is $0.34 \mathrm{THz}$, system transmission power is respectively selected for $10 \mathrm{~mW}$ and $1 \mathrm{~W}$, bandwidth is $50 \mathrm{GHz}$. Known from the figure, when the antenna gain is more than $40 \mathrm{~dB}$, the system's maximum communication capacity increases significantly. The antenna gain raises more than $50 \mathrm{~dB}$, communication capacity of $1 \mathrm{~W}$ transmitted power is higher $70 \mathrm{Gbps}$ than that of $10 \mathrm{~mW}$. When the system communication bandwidth increases from $10 \mathrm{G}$ to $50 \mathrm{G}$, the system largest communication capacity increases significantly, as shown in Fig.2.

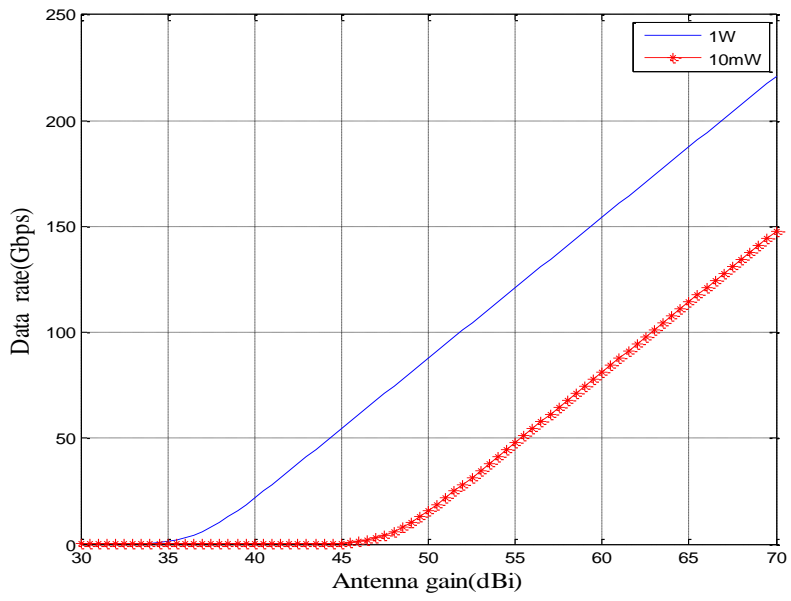

Figure 1. The maximum capacity of short distance communication system.

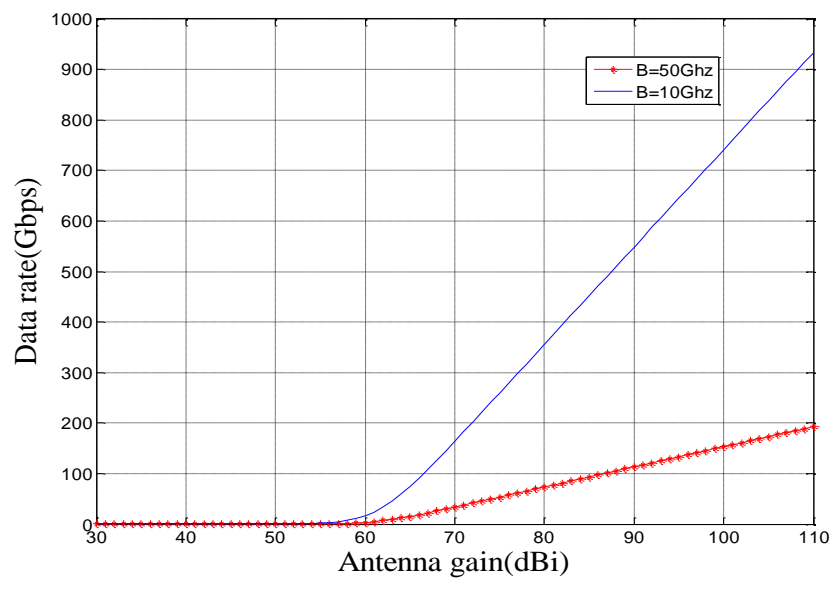

Figure 2 The maximum capacity of short distance communication system under different bandwidth
Based on the design given by [14], the antenna gain is closely related to the aperture size. As shown in Fig.3, when frequency is $0.34 \mathrm{THz}$ and gain is $70 \mathrm{~dB}$, antenna needs $1.2 \mathrm{~m}$ aperture. Then, according to Fig.1 and Fig.3, to achieve $100 \mathrm{Gbps}$ or higher communication capacity, it requires $10 \mathrm{~mW}$ system transmission power, $65 \mathrm{~dB}$ antenna gain and $68 \mathrm{~cm}$ antenna aperture. If the channel bandwidth increases to $50 \mathrm{GHz}$, it can get higher communication capacity more than 500Gbps. The traditional microwave frequency wireless communication system is impossible to achieve.

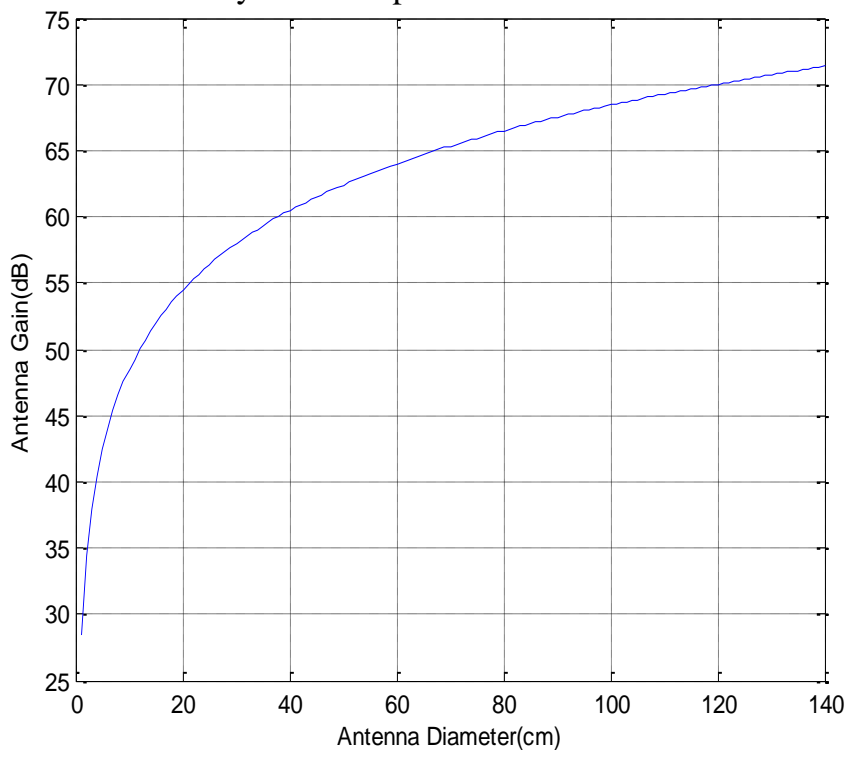

Figure 3. The relationship between antenna gain and aperture size

\section{B. Long Range Satellite Communication}

In the Fig.4, communication distance is $100 \mathrm{~km}$, it analyzes different transmission power relationship between communication capacity and antenna gain. As shown in Fig.4 (a), when system transmission power is $10 \mathrm{~mW}$, reaching $2.5 \mathrm{Gbps}$ or higher communication capacity needs antenna gain increased to $71 \mathrm{~dB}$, then the corresponding antenna aperture is $120 \mathrm{~cm}$. As shown in Fig.4 (b), if the system transmission power can be increased to $1 \mathrm{~W}$, it only needs to design a antenna of $62 \mathrm{~dB}$ gain, $50 \mathrm{~cm}$ aperture and can reach $2.5 \mathrm{Gbps}$ or higher communication capacity.

Combining the existing antenna design, the paper analyzes inter-satellite communication capacity between short distance of $1 \mathrm{~km}$ and long distance of $100 \mathrm{~km}$. The results show that in order to realize the short distance $0.34 \mathrm{THz}$ ultra-fast communication application, system design has low requirements to the antenna size, but it has higher demands for long range communication antenna. For example, the bandwidth is the premise, in the short range communication, to reach $100 \mathrm{Gbps}$ or higher data transmission rate, it requires $10 \mathrm{~mW}$ system transmission power, $65 \mathrm{~dB}$ antenna gain, $68 \mathrm{~cm}$ aperture. To achieve $2.5 \mathrm{Gbps}, \quad 100 \mathrm{~km}$ long rangeinter-satellite terahertz communication, it needs to design $62 \mathrm{~dB}$ gain and $50 \mathrm{~cm}$ antenna aperture, and demands that the system transmission power reaches $1 \mathrm{~W}$. Therefore, long distance and large capacity are feasible for terahertz satellite communication. 


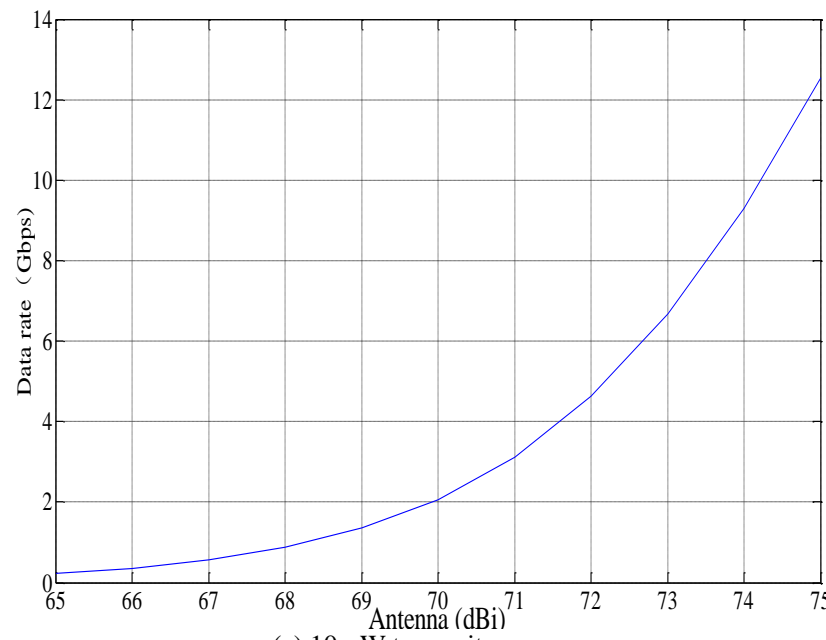

(a) $10 \mathrm{~mW}$ transmit power

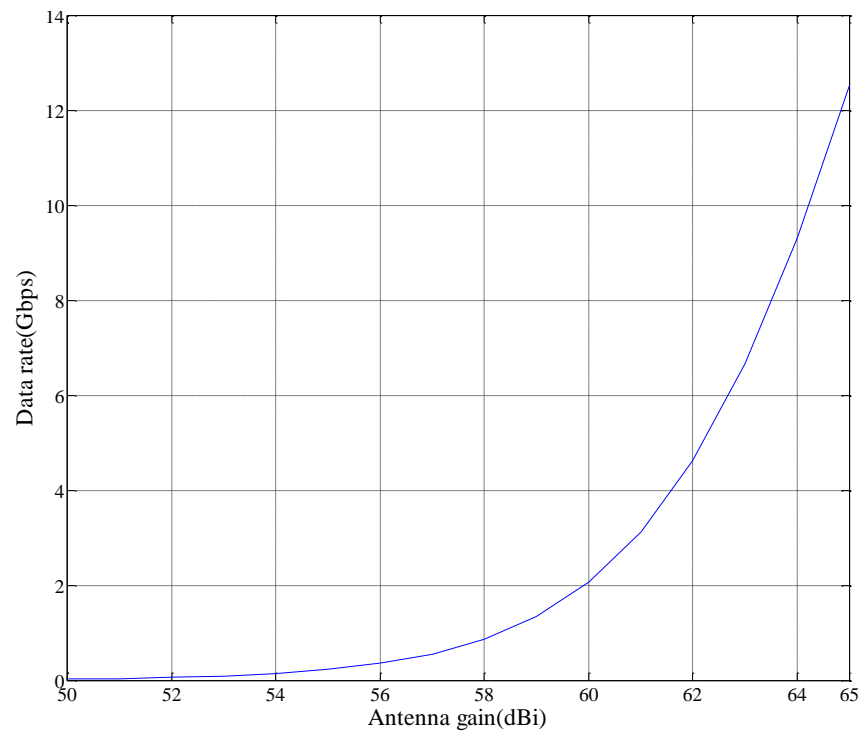

(b) $1 \mathrm{~W}$ transmit power

Figure 4. The relationship between the communication capacity of different transmit power and antenna gain $(100 \mathrm{~km}$ long distance communication).

\section{CONCLUSION}

Combining the current status of the development of antenna technology, this paper analyzes the feasibility of ultra high speed short distance and high speed long distance inter-satellite terahertz communication. The results show that the short distance between satellites within $1 \mathrm{~km}$ and $0.34 \mathrm{THz}$ communication applications for antenna size requirement is lower, but has the higher demand for long distance communication antenna. To reach $2.5 \mathrm{Gbps}, 100 \mathrm{~km}$ inter-satellite terahertz communication, it needs to design $62 \mathrm{~dB}$ gain, $50 \mathrm{~cm}$ antenna aperture, and demands that the system transmission power reaches $1 \mathrm{~W}$. Distributed clusters terahertz communication application system, to realize the technology advantage of high speed, long distance, large capacity, demands high gain antenna, high power transmission system and the guarantee of APT system. Therefore, as the future research, it is necessary to analyze the demand for APT technology and satellite platform vibration influence on inter-satellite terahertz communication, further design inhibiting platform vibration feedback control system.

\section{ACKNOWLEDGMENT}

This work was supported by the National Natural Science Foundation of China (61540024), and the Program for Liaoning Innovative Research Team in University (LT2014025) and the Liaoning Natural Science Foundation (No. 2014020131).

\section{REFERENCES}

[1] H. J. Song and K. Ajito, "24Gbit/s data transmission in $300 \mathrm{GHz}$ band for futureterahertz communication," Electronics Letters,vol. 48, no. 7, pp. 31-37, 2012.

[2] H. Song and T. Nagatsuma, "Present and future of Terahertz communications," IEEE Transaction on Terahertz Science and Technology, vol. 1, no. 9, pp. 256-263, 2011.

[3] J. Antes, J. Reicharty, and D. Lopez-Diaz, "System concept and implementation of a $\mathrm{mmW}$ wireless link providing data rates up to 25Gbit/s," Electronics Letters, vol. 49, no. 9, pp. 34-40, 2013.

[4] X. Deng, C. Wang, C. Ling, et al., " $0.14 \mathrm{THz}$ ultra high speed wireless communication system experiment research," High Power Laser and Particle Beam, vol. 23, no. 6, pp. 1430-1432, 2011.

[5] C. Wang, B. Lu, L. Miu, et al., " $0.34 \mathrm{THz}$ wireless communication transceiver front end," High Power Laser and Particle Beams, vol. 25, no.6, pp. 1530-1534, 2013.

[6] G. Ducournau, P. Szriftgiser, and J. F Lampin,"Waveguide-based electronic Tx/Rx for THz range applications," Proc.16th International Radar Symposium (IRS), 2015, pp. 24-26.

[7] C. Wang, J. Liu, S. Wu,et al., " $0.14 \mathrm{THz} 10 \mathrm{Gbps}$ wireless communication system," Information and Electronic Engineering, no.3, pp. 265-269, 2011.

[8] K. C. Jastrow, "Measurement of channel and propagation properties at 300GHz,” IEEE Spectrum, vol. 41, no. 7, pp. 1324-1329, 2012.

[9] Y. Lu, "Research of terahertz technology in space field," Infrared, vol.36, no.1, pp.1-11, 2015.

[10] L. Song and Y.Ding, "THz space communication system transmission characteristics research," Computer simulation, vol. 32, no. 7, pp.194-197, 2015.

[11] C. Pan,W. Dong, and Y. Ding, "Modeling and BER characteristics analysis of inter-satellite THz communication links," ICIC Express Letters, vol. 9, no. 6, pp. 1597-1602, 2015.

[12] Z. Hao and W. Zhang, "Preliminary scheme design of the inter satellite terahertz communication transmission system," Radio Communication Technology, no. 3, pp. 32-34, 2012.

[13] T. Zhang, Q. Fu, et al.,"Design of a common focus reflective optical antenna for multi platform laser communication," Acta Optica Sinica, vol. 35 , no. 10 , pp. 2-5, 2015.

[14] L. Shang, Z. Xia, and X. Liao, "Influence of structure and parameters on gain of small aperture THz," Infrared, vol. 32, no. 2, pp. 38-42, 2011.

[15] C. Pan, L. Yang, C. Liu, and Y. Xie, "BLMS transversal filter-based control module for vibration compensation," Chinese patent, 2012205429039, 2012-10-22. 